\title{
Aspartate aminotransferase to platelet ratio index and sustained virologic response are associated with progression from hepatitis $C$ associated liver cirrhosis to hepatocellular carcinoma after treatment with pegylated interferon plus ribavirin
}

This article was published in the following Dove Press journal:

Clinical Interventions in Aging

I August 2016

Number of times this article has been viewed

Khai-Jing Ng, ${ }^{1,2, *}$ Chih-Wei

Tseng, ${ }^{1-4, *}$ Ting-Tsung Chang, ${ }^{5,6}$ Shinn-Jia Tzeng, ${ }^{7}$

Yu-Hsi Hsieh, ${ }^{1,2}$ Tsung-Hsing Hung, ${ }^{1,2}$ Hsiang-Ting Huang, ${ }^{8}$ Shu-Fen Wu, ${ }^{9}$ Kuo-Chih

Tseng ${ }^{1,2}$

'Department of Internal Medicine, Dalin Tzu Chi Hospital, Buddhist Tzu Chi Medical Foundation, Chia-Yi,

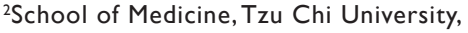
Hualien, ${ }^{3}$ Division of Gastroenterology, Department of Medicine, Taipei Veterans General Hospital, Taipei, ${ }^{4}$ School of Medicine, National Yang-Ming University, Taipei, ${ }^{5}$ Department of Internal Medicine, National Cheng Kung University Medical College and Hospital, Tainan, ${ }^{6}$ Infectious Disease and Signaling Research Center, National Cheng Kung University, Tainan, ${ }^{7}$ Department of Agronomy, National Chiayi University, Chia-Yi, ${ }^{8}$ Department of Nursing, Dalin Tzu Chi Hospital, Buddhist Tzu Chi Medical Foundation, Chia-Yi, ' Institute of Molecular Biology, National Chung Cheng University, Chia-Yi, Taiwan

*These authors contributed equally to this work

Correspondence: Kuo-Chih Tseng Department of Internal Medicine, Dalin Tzu Chi Hospital, Buddhist Tzu Chi Medical Foundation, No 2, Ming-Shen Road, Dalin

Town, Chia-Yi County 622, Taiwan

Tel +8865264 8000 ext 524l

Fax +88652648006

Email tsengkuochih@gmail.com
Background: The aim of this study was to evaluate the clinically significant predictors of hepatocellular carcinoma ( $\mathrm{HCC}$ ) development among hepatitis $\mathrm{C}$ virus ( $\mathrm{HCV}$ ) cirrhotic patients receiving combination therapy.

Patients and methods: One hundred and five compensated cirrhosis patients who received pegylated interferon plus ribavirin between January 2005 and December 2011 were enrolled. All the patients were examined with abdominal sonography and liver biochemistry at baseline, end of treatment, and every 3-6 months posttreatment. The occurrence of HCC was evaluated every 3-6 months posttreatment.

Results: A total of 105 patients were enrolled (mean age $58.3 \pm 10.4$ years). The average follow-up time for each patient was 4.38 years (standard deviation 1.73 years; range 1.13-9.27 years). Fifteen (14.3\%) patients developed HCC during follow-up period. Thirteen of them had high baseline aspartate aminotransferase to platelet ratio index (APRI) (ie, an APRI > 2.0). Multivariate analysis showed that those without sustained virologic response (SVR) (hazard ratio [HR] 5.795; 95\% confidence interval [CI] 1.370-24.5; $P=0.017$ ) and high APRI (HR 5.548; 95\% CI 1.191-25.86; $P=0.029$ ) had a significantly higher risk of HCC occurrence. The cumulative incidence of HCC was significantly higher $(P=0.009)$ in patients without SVR (3-year cumulative incidence $21.4 \%$; 95\% CI 7.4\%-35.5\%; 5-year cumulative incidence $31.1 \%$; 95\% CI $11.2 \%-51.1 \%$ ) compared to those with SVR (3- and 5-year cumulative incidence $6.2 \%$; 95\% CI $0 \%-1.3 \%)$. Further, the cumulative incidence of HCC was significantly higher $(P=0.006)$ in patients with high APRI (3-year cumulative incidence 21.8\%; 95\% CI 8.2\%-35.3\%; 5-year cumulative incidence $30.5 \%, 95 \%$ CI $11.8 \%-49.3 \%$ ) compared to those with low APRI (3- and 5-year cumulative incidence $4.2 \%, 95 \%$ CI $0 \%-1.0 \%$ ).

Conclusion: In HCV-infected cirrhotic patients who received combination therapy, APRI and SVR are the two major predictors of HCC development.

Keywords: aspartate aminotransferase to platelet ratio index, chronic hepatitis $\mathrm{C}$, hepatitis $\mathrm{C}$ virus, hepatocellular carcinoma, liver cirrhosis, sustained virologic response

\section{Introduction}

Hepatocellular carcinoma (HCC) is one of the most deadly cancers and is the third leading cause of cancer-related death among males and the sixth among females worldwide. ${ }^{1}$ According to a recent national analysis in Taiwan, HCC remains the second 
leading cause of cancer-related death among both males and females during the past 10 years. ${ }^{2}$ Chronic hepatitis is one of the most important causes of chronic liver diseases, including cirrhosis that can lead to subsequent decompensation and development of HCC. ${ }^{3}$

The estimated risk of HCC is 15-20 times higher among persons infected with hepatitis $\mathrm{C}$ virus (HCV) compared to those without infection, and the greatest excess risk occurs in those with advanced hepatic fibrosis or cirrhosis. ${ }^{4,5}$ In chronic HCV antiviral therapy, a sustained viral response (SVR) had been a clinically meaningful end point at which viral clearance contributes to reduced inflammation and histologically identified fibrosis, fewer hepatic complications, lower liverrelated mortality, and a reduced incidence of HCC. ${ }^{6-8}$ Even in patients with advanced liver disease, SVR has been found to be associated with HCC risk reduction. ${ }^{9}$

Risk factors for HCV-related HCC include older age, male sex, coinfection with human immunodeficiency virus (HIV) or hepatitis B virus (HBV), obesity, hepatic fibrosis, alcohol abuse, and sex hormone dysregulation. .,10,11 $^{\text {The liver }}$ fibrosis stage provides important prognostic information about the development of HCC. The aspartate aminotransferase to platelet ratio index (APRI) is a noninvasive marker that has been validated for the diagnosis of both significant fibrosis and cirrhosis. ${ }^{12,13}$ APRI is a useful marker for the prognosis in chronic hepatitis $\mathrm{C}(\mathrm{CHC})$ patients. ${ }^{14}$ Some recent studies report that the APRI score could be a predictor of HCC in chronic hepatitis patients. ${ }^{15,16}$ APRI could be a useful marker to classify HCC risk in CHC patients who achieved SVR and predict $\mathrm{HCC}$ recurrence after radiofrequency ablation. ${ }^{17-19}$ However, the prognostic value of APRI in cirrhotic patients for predicting the occurrence of HCC is uncertain.

Hence, the aim of this study was to evaluate the clinically significant predictors of HCC development among HCV cirrhotic patients. These factors may affect physicians' clinical decisions.

\section{Patients and methods Selection of patients}

HCV patients with Child A cirrhosis without decompensation and who underwent treatment at the Dalin Tzu Chi General Hospital with either pegylated interferon (PEG-IFN)- $\alpha$-2a or PEG-IFN- $\alpha$-2b plus ribavirin (RBV) between January 2005 and December 2011 were enrolled in this retrospective study. All the patients were positive for antihepatitis $\mathrm{C}$ antibody for more than 6 months, had an alanine aminotransferase (ALT) level higher than the upper limit of normal, and had detectable serum HCV RNA. We excluded patients with posttreatment follow-up $<1$ year, HCC history, incomplete medical records, autoimmune diseases, HIV infection, neutropenia $(<1,500$ neutrophils/mL), thrombocytopenia $(<50,000$ platelets/mL), anemia $(<12 \mathrm{~g}$ of hemoglobin/dL in females and $<13 \mathrm{~g} / \mathrm{dL}$ in males), or poorly controlled psychiatric disease.

One hundred and twenty compensated cirrhosis patients who received PEG-IFN plus RBV were initially enrolled (Figure 1). Five patients had posttreatment follow-up time $<1$ year, seven did not have complete medical records, and three previously had HCC, and all these patients were excluded from the study. The remaining 105 patients were finally included. The average follow-up time for each patient was 4.38 years (standard deviation [SD] 1.73 years; range 1.13-9.27 years). The study was approved by the Research Ethics Committee of Dalin Tzu Chi Hospital (B099010124). Written informed consent was obtained from each patient, and the Research Ethics Committee of Dalin Tzu Chi Hospital approved the written consent process.

\section{Regimen of PEG-IFN plus RBV}

PEG-IFN- $\alpha-2$ a (Pegasys; Hoffman-La Roche Ltd., Basel, Switzerland) or PEG-IFN- $\alpha-2 b$ (PegIntron; ScheringPlough Corp., Kenilworth, NJ, USA) plus RBV were prescribed to eligible patients for 6 months. PEG-IFN- $\alpha-2 \mathrm{a}$ $(180 \mu \mathrm{g} / \mathrm{kg})$ or PEG-IFN- $\alpha-2 \mathrm{~b}(1.5 \mu \mathrm{g} / \mathrm{kg})$ was administered once per week by subcutaneous injection. The fixed duration (6 months) without consideration of HCV genotypes is due to restrictions imposed by the reimbursement policy of the Bureau of National Health Insurance in Taiwan.

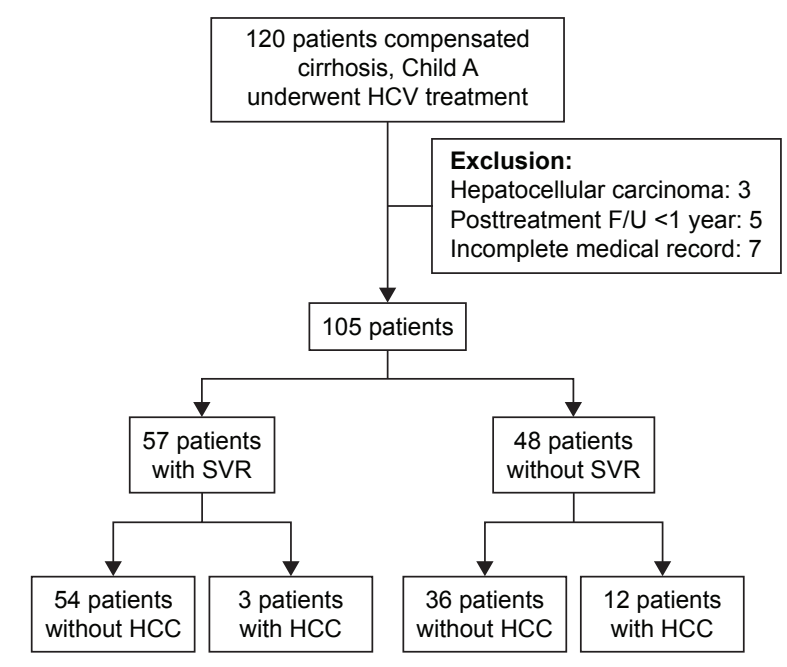

Figure I Flow diagram representing the study cohort.

Note: Dalin Tzu Chi Hospital, January 2005-December 201 I.

Abbreviations: HCV, hepatitis C virus; F/U, follow-up; SVR, sustained virologic response; HCC, hepatocellular carcinoma. 
RBV was prescribed orally at a dose of $800 \mathrm{mg} /$ day for patients $<55 \mathrm{~kg}, 1,000 \mathrm{mg} /$ day for patients between 55 and $75 \mathrm{~kg}$, and 1,200 mg/day for patients $>75 \mathrm{~kg}$. Adjustments to the dose of PEG-IFN and RBV and treatment with either erythropoietin or blood transfusion were determined according to published practice guidelines, ${ }^{3,20-22}$

\section{Clinical monitoring}

The primary outcome was time to develop HCC. All patients were examined at baseline, end of treatment, and every 3-6 months posttreatment with a liver function test and abdominal sonography at the hospital's gastrointestinal outpatient clinic. The serum aspartate aminotransferase (AST), ALT, total bilirubin, creatinine, hemoglobin, white blood cell count, and platelet count were measured at each time point. HCV RNA was quantified at baseline and 24 weeks posttreatment. The diagnosis of liver cirrhosis was made by liver biopsy or by clinical diagnostic criteria including twice documented ultrasonographic evidence of a coarse and nodular parenchyma, irregular surface, and dull margin with splenomegaly, ascites, hepatic encephalopathy, or varices. ${ }^{23}$ Liver biopsy with optional procedure was performed at baseline with patients' consent. Liver biopsy was obtained from 62 patients (59.6\%) in this study. A diagnosis of fatty liver was based on the results of biopsy and/or abdominal ultrasound. Other clinical factors, including diabetes mellitus, chronic hepatitis $\mathrm{B}$ (CHB), and alcoholism, were also evaluated by chart review. The diagnosis of CHB was based on seropositive status for hepatitis B surface antigen for at least 6 months. HCC was diagnosed either by biopsy or by imaging in the setting of liver cirrhosis. The specific imaging pattern was defined by increased contrast uptake in the arterial phase followed by contrast washout in the venous/delayed phase as seen via computer tomography or magnetic resonance. ${ }^{24,25}$ In this study, we calculated APRI using the formula of ([AST/upper limit of normal]/platelet count $\left.\left[10^{9} / \mathrm{L}\right]\right) \times 100$, and further used it as a noninvasive marker validated for the diagnosis of both significant fibrosis and cirrhosis. The cutoff value of APRI $>2.0$ defined high APRI and APRI $<2.0$ defined low APRI. ${ }^{3,12,13}$

\section{HCV quantification and genotyping}

Serum HCV RNA was quantified at baseline and 24 weeks posttreatment using real-time polymerase chain reaction with a detection limitation of $15 \mathrm{IU} / \mathrm{mL} .{ }^{26}$ The threshold for discriminating low- and high-baseline HCV RNA was 400,000 IU/mL. ${ }^{22} \mathrm{HCV}$ genotyping was performed using melting curve analysis (Roche LightCycler; Biotronics Tech Corp., Lowell, MA, USA). ${ }^{27}$

\section{Sustained virologic response}

SVR was defined as an undetectable HCV RNA for at least 24 weeks after the patient completed the combined treatment of PEG-IFN plus RBV. Patients who were positive for HCV RNA at week 24 posttreatment were considered non-SVR.

\section{Statistical analysis}

SPSS 19.0 for Windows (IBM Corporation, Armonk, NY, USA) was used for all statistical analyses. The chi-square test or the Fisher's exact test was used for nominal variables. Student's $t$-test was used to compare continuous variables with normal distributions, and the Mann-Whitney $U$-test was used for continuous variables with nonnormal distributions. In competing risk data ratios, we conducted calculations and comparisons of cumulative incidences using a modified Kaplan-Meier method. We tested differences in the full time-to-event distributions between the study groups using a log-rank test. To determine the independent risk factors for HCC occurrence, we carried out multivariate analyses and stratified analyses using HRs with Cox proportional hazards regression models in the presence of a competing risk event after adjusting for age, sex, high APRI, SVR, genotype, and cohepatitis (including hepatitis B coinfection, alcoholism, or fatty liver). Results were shown as HRs with 95\% CIs. Significances in all analyses were set as $P<0.05$.

\section{Results \\ General characteristics of the patients}

A total of 105 patients with $\mathrm{HCV}$-associated Child A cirrhosis without decompensation were enrolled, consisting of 57 patients $(54.3 \%)$ with SVR and 48 patients without SVR $(45.7 \%)$ by intention-to-treat analysis. Of the 105 patients, $66(62.9 \%)$ were infected with HCV genotype 1, and the remaining 39 patients (37.1\%) were infected with other genotypes of HCV. There were 47 males and 58 females, and the mean patient age was $58.3 \pm 10.4$ years. Thirteen patients $(12.4 \%)$ did not complete the full treatment regimen (mean treatment duration, 61 days). One patient (1/13) stopped treatment due to major trauma with renal hemorrhage. Twelve patients (12/13, 92.3\%) did not accept complete treatment due to treatment side effects. Among those patients, decompensation with jaundice (4/13) was the most common reason. An intention-to-treat analysis was performed in this study. No deaths or severe treatmentrelated complications were found during the course of treatment. The average follow-up time for each patient was 4.38 years (SD 1.73 years; range 1.13-9.27 years) (Table 1 ). Fifteen (14.3\%) patients developed HCC during follow-up 
Table I Baseline characteristics and treatment outcomes between patients with and without progression to HCC

\begin{tabular}{|c|c|c|c|c|}
\hline Variable & $\begin{array}{l}\text { Total } \\
(n=105)\end{array}$ & $\begin{array}{l}\text { Without progression } \\
\text { to HCC }(n=90)\end{array}$ & $\begin{array}{l}\text { Progression to } \\
\text { HCC }(n=\mid 5)\end{array}$ & $P$-value \\
\hline Follow-up time, years ${ }^{\mathrm{a}}$ & $4.4 \pm 1.7$ & $4.4 \pm I .7$ & $4.8 \pm 2.0$ & 0.392 \\
\hline Age, years ${ }^{\mathrm{a}}$ & $58.3 \pm 10.4$ & $57.4 \pm 10.7$ & $63.5 \pm 5.7$ & $0.028 *$ \\
\hline Male, n (\%) & $47(44.8)$ & $4 I(45.6)$ & $6(40.0)$ & 0.783 \\
\hline SVR, n (\%) & $57(54.3)$ & $54(60.0)$ & $3(20.0)$ & $0.005^{*}$ \\
\hline DM, n (\%) & $28(26.7)$ & $24(26.7)$ & $4(26.7)$ & 1.000 \\
\hline Cohepatitis, n (\%) & $51(48.6)$ & $46(5 I .1)$ & $5(33.3)$ & 0.268 \\
\hline HBV coinfection, n (\%) & $10(9.5)$ & $9(10)$ & I (6.7) & 1.000 \\
\hline Fatty liver, n (\%) & 44 (4I.9) & $39(43.3)$ & $5(33.3)$ & 0.577 \\
\hline Alcoholism, n (\%) & $10(9.5)$ & $10(11.1)$ & $0(0)$ & 0.351 \\
\hline Genotype I, n (\%) & 66 (62.9) & $57(86.4)$ & $9(13.6)$ & 0.782 \\
\hline High RNA load, n (\%) & $76(72.4)$ & $66(73.3)$ & $10(66.7)$ & 0.756 \\
\hline $\mathrm{APRI}^{\mathrm{a}}$ & $2.4 \pm 1.6$ & $2.2 \pm 1.4$ & $4.1 \pm 2.0$ & $0.001 *$ \\
\hline $\mathrm{ALT}^{\mathrm{a}}$ & $122.9 \pm 8 \mid .7$ & $117.9 \pm 84.3$ & $153.3 \pm 57.2$ & $0.014 *$ \\
\hline $\mathrm{AST}^{\mathrm{a}}$ & $93.2 \pm 51.4$ & $87.6 \pm 50.3$ & $127.0 \pm 46.5$ & $0.002 *$ \\
\hline Platelet $^{\mathrm{a}}$ & $124.4 \pm 49.2$ & $129.1 \pm 50.9$ & $96.5 \pm 22.8$ & $0.017 *$ \\
\hline $\mathrm{AFP}^{\mathrm{a}}$ & $16.6 \pm 23.2$ & $16.2 \pm 23.9$ & $19.0 \pm 18.9$ & 0.233 \\
\hline Albumin $^{a}$ & $4.0 \pm 0.4$ & $4.0 \pm 0.4$ & $3.9 \pm 0.3$ & 0.179 \\
\hline $\mathrm{PT} / \mathrm{INR}^{\mathrm{a}}$ & $I . I \pm 0.1$ & $1.1 \pm 0.1$ & $1.1 \pm 0.1$ & 0.762 \\
\hline Total bilirubin ${ }^{a}$ & $0.9 \pm 0.4$ & $0.9 \pm 0.3$ & $1.1 \pm 0.5$ & 0.182 \\
\hline
\end{tabular}

Notes: ${ }^{a}$ Data are expressed as mean \pm standard deviation; ${ }^{b}$ cohepatitis includes hepatitis B coinfection, alcoholism, or fatty liver; ' the cutoff value for discriminating low and high baseline HCV RNA is $400,000 \mathrm{IU} / \mathrm{mL}$. Student's $t$-test and the Mann-Whitney U-test were used to compare continuous variables. The Fisher's exact test was used to compare the categorical variables. $* P<0.05$.

Abbreviations: SVR, sustained virologic response; DM, diabetes mellitus; APRI, aspartate aminotransferase to platelet ratio index; HCC, hepatocellular carcinoma; HBV, hepatitis B virus; PT/INR, international normalized ratio of prothrombin time; AFP, alpha-fetoprotein; ALT, alanine aminotransferase; AST, aspartate aminotransferase; $\mathrm{HCV}$, hepatitis $\mathrm{C}$ virus.

period; 13 in this group had high baseline APRI $(>2.0)$ and two had low APRI $(<2.0)$.

\section{Demographics and clinical features that predispose to HCC}

Table 1 shows the baseline characteristics and treatment outcomes of the patients with and without progression to HCC. We performed univariate analyses on the two groups and found significant differences in age, SVR, baseline APRI, platelet count, AST, and ALT. No significant differences in sex, diabetes, cohepatitis (alcoholism, HBV coinfection, or fatty liver), HCV genotype, HCV RNA load, alpha-fetoprotein, albumin, international normalized ratio of prothrombin time, and total bilirubin were found between the two groups.

\section{Multivariate stratified analysis}

Table 2 shows the results of the Cox regression analysis. After adjustments for age, sex, high APRI, SVR, genotype, and cohepatitis (including hepatitis B coinfection, alcoholism, or fatty liver), those without SVR (HR 5.795; 95\% CI $1.370-24.5 ; P=0.017)$ and those with a high APRI level (HR 5.548; 95\% CI 1.191-25.86; $P=0.029$ ) were associated with a significantly higher risk of HCC occurrence.

\section{Cumulative incidences of HCC occurrence}

The cumulative incidences of HCC among patients with and without SVR after treatment are shown in Figure 2. The cumulative incidence of HCC was significantly higher $(P=0.009)$ in patients without SVR (3-year cumulative incidence 21.4\%; $95 \%$ CI 7.4\%-35.5\%; 5-year cumulative incidence $31.1 \%$; $95 \%$ CI $11.2 \%-51.1 \%$ ) compared to those with SVR (3- and 5-year cumulative incidence $6.2 \% ; 95 \% \mathrm{CI} 0 \%-1.3 \%$ ). The difference in 5-year cumulative incidence was $24.9 \%$. The unadjusted number needed to treat associated with 1 less HCC within 5 years was 4, suggesting that four patients who achieved SVR were associated with 1 less HCC within 5 years of treatment.

Table 2 Adjusted HRs for HCC progression with Cox regression analysis*

\begin{tabular}{llll}
\hline Variable & HR & $\mathbf{9 5 \%} \mathbf{C l}$ & P-value \\
\hline SVR & 5.795 & $1.37-24.52$ & $0.017^{*}$ \\
Age, years & 1.041 & $0.98-1.11$ & 0.210 \\
Male & 0.772 & $0.25-2.38$ & 0.653 \\
High APRI $^{a}$ & 5.549 & $1.19-25.86$ & $0.029^{*}$ \\
\hline
\end{tabular}

Notes: ${ }^{a}$ The cutoff value for discriminating low and high APRI is $2 ;{ }^{*} P<0.05$. Abbreviations: HR, hazard ratio; HCC, hepatocellular carcinoma; SVR, sustained virologic response; $\mathrm{Cl}$, confidence interval; $\mathrm{APRI}$, aspartate aminotransferase to platelet ratio index. 


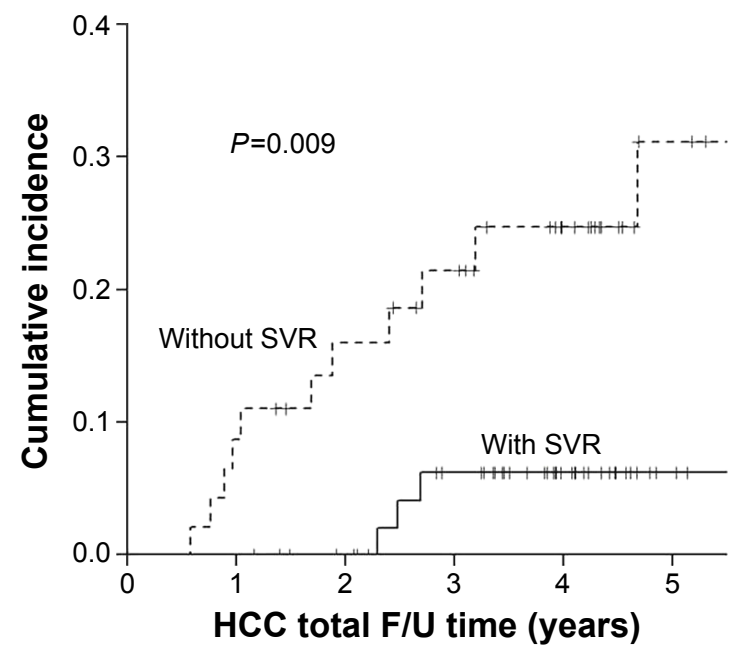

\section{No at risk}

Without SVR 48

With SVR 57

$\begin{array}{lllll}44 & 39 & 35 & 25 & 14 \\ 57 & 53 & 45 & 29 & 14\end{array}$

Figure 2 Cumulative risk of hepatocellular carcinoma with and without SVR. Abbreviations: F/U, follow-up; SVR, sustained virologic response; HCC, hepatocellular carcinoma.

Figure 3 shows the cumulative incidence among patients with high and low APRI values, based on a cutoff value of 2.0. The cumulative incidence of HCC was significantly higher $(P=0.006)$ in patients with a high APRI value (3-year cumulative incidence $21.8 \%$; 95\% CI 8.2\%-35.3\%; 5-year cumulative incidence $30.5 \%$; 95\% CI $11.8 \%-49.3 \%$ ) compared to those with low APRI values (3- and 5-year

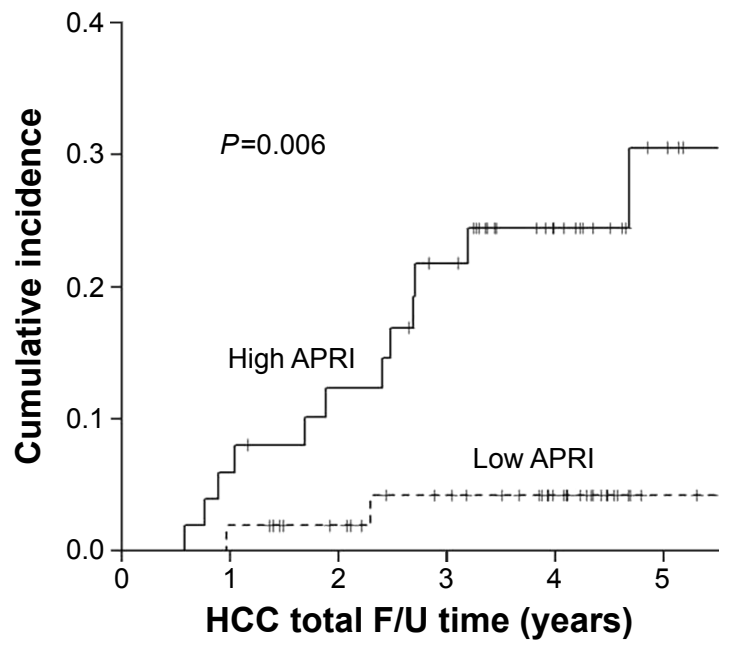

No at risk

$\begin{array}{lllllll}\text { Low APRI } & 53 & 52 & 47 & 41 & 29 & 13\end{array}$

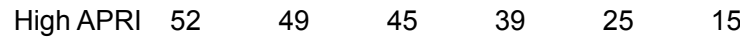

Figure 3 Cumulative risk of hepatocellular carcinoma with high and low APRI. Note: The cutoff value for discriminating low and high APRI is 2.

Abbreviations: APRI, aspartate aminotransferase to platelet ratio index; F/U, followup; HCC, hepatocellular carcinoma. cumulative incidence $4.2 \%$; $95 \%$ CI $0 \%-1.0 \%$ ). The difference in 5 -year cumulative incidences was $26.3 \%$.

\section{Discussion}

$\mathrm{HCV}$ is a single-stranded RNA virus, and its genome is never integrated into the genome of hepatocytes. Although no known oncogenic properties have been reported with respect to $\mathrm{HCV}$, the multiple functions of $\mathrm{HCV}$ proteins and their effects on the modulation of intracellular signaling transduction processes may facilitate carcinogenesis via interactions of viral proteins with host cell proteins. ${ }^{28} \mathrm{HCV}$ core proteins lead to the promotion of cellular transformation, and HCV-related chronic inflammation has been responsible for promoting mutations during hepatocyte regeneration, thereby contributing to HCC development. ${ }^{29,30}$ Hepatocyte regeneration and cycling that occur after antiviral therapy may activate cellular pathways involved in the development of dysplasia, increasing the risk of hepatocarcinogenesis. ${ }^{31}$ Interferon-based therapy was found to reduce the risk of HCC development, particularly in virologic or biochemical responders. ${ }^{32-34}$ Despite the larger magnitude of risk reduction in patients with SVR, there remains a definite risk of HCC in SVR patients. ${ }^{11,35}$ The risk factors for HCV-related HCC include older age, male, coinfection with HIV or HBV, obesity, hepatic fibrosis, alcohol abuse, and sex hormone dysregulation. ${ }^{4,10,11}$

A meta-analysis summarized the evidence from 30 observational studies that examined the risk of HCC among HCV-infected patients and found that SVR was associated with a reduction in the relative risk of $\mathrm{HCC}$ for patients at all stages of liver disease (HR $0.24 ; 95 \%$ CI $0.18 \%-0.31 \%$; $P<0.001)$ and a higher absolute benefit (HCC prevention) in patients with advanced liver disease who achieved SVR. ${ }^{9}$ Similarly, in our study, HCC development in cirrhotic HCV patients was significantly lower in the SVR group. Those patients without SVR (HR 5.795; 95\% CI 1.370-24.5; $P=0.017$ ) were associated with a significantly higher risk of HCC occurrence. A large cohort study conducted in Taiwan showed similar results in $\mathrm{HCC}$ risk reduction among cirrhotic HCV patients. ${ }^{10}$

Notably, our study results found that high APRI $(>2)$ is an independent risk factor for HCC development (HR 5.548; $95 \%$ CI 1.191-25.86; $P=0.029)$. APRI is a noninvasive measurement for diagnosing hepatic fibrosis and cirrhosis and was recommended by the World Health Organization (WHO) guidelines for HCV infection published in 2014. ${ }^{3}$ Based on the WHO recommendation, patients with values above the high APRI cutoff value $(>2)$ should be treated with higher priority 
because they have a high probability of developing F4 cirrhosis. $^{3}$ A recent study showed that a higher APRI significantly increased the risk of $\mathrm{HCC}$ in cirrhotic $\mathrm{HBV}$-infected patients, but this finding was attenuated after multivariate adjustment. ${ }^{16}$ More recently, in a cohort of 642 SVR Taiwan patients $(13 \%$ cirrhotics), HCC was strongly associated with cirrhosis (HR 4.98; 95\% CI 2.32-10.71; $P<0.001)$ and less strongly with age (HR 1.06; 95\% CI 1.02-1.11; $P=0.005$ ) and Gammaglutamyl transpeptidase ( $\gamma$ GT) (HR 1.01; 95\% CI 1.00-1.013; $P<0.001) .{ }^{36}$ This study supports the importance of cirrhosis as the predictor of $\mathrm{HCC}$ in $\mathrm{CHC}$ patients and potential role of APRI. As far as we know, few studies have evaluated the possible relationship between APRI and HCC development in HCV-infected patients after SVR. ${ }^{15,17,18}$ Our study included pure cirrhotic cohort and showed that high APRI $(>2.0)$ is significantly related to HCC development even after multivariate adjustment. The data from recent cohort support the recommendation that a higher treatment priority should be given to those patients who present with a high APRI. ${ }^{3}$

Although a high proportion of genotype $1(62 \%)$ and advanced liver disease with short treatment duration (6 months) cause lower SVR rate (54.3\%), a recent study showed the achievement of SVR could slow down the rapid progression of HCC among cirrhotic patients. ${ }^{9}$ In the era of new, interferonsparing, antiviral strategies, the SVR rate for similar patients is more than $90 \%$. The results of our study support the significant benefit of SVR achievement in lowering the progression of HCC, and the APRI score could help us identify patients at higher risk of HCC development. The direct antiviral agent treatment should be provided for the cirrhotic patients even though it is expensive.

Our study found no difference between 3- and 5-year cumulative incidences of $\mathrm{HCC}$ in patients with SVR or with low APRI, as shown in Figures 2 and 3. The stabilization of cumulative incidences may indicate that HCC development is more closely related to developments during the first few years after SVR achievement in low APRI patients. The interaction between SVR and APRI appears to be complicated. A higher fibrosis stage is associated with lower SVR rate, and typically the fibrosis stage improves after SVR is achieved. This may be due to high SVR durability with a low relapse rate, which has been confirmed in HCV-infected liver transplant recipients, hemophiliacs, cirrhotics, those coinfected with HIV, and children. A durable SVR may improve the fibrosis stage and decrease the incidence of HCC 3 years later. Hence, HCV cirrhotic patients (especially those with a high APRI) should be treated as early as possible because of potential beneficial effects in HCC prevention.

There are some limitations in our study. First, liver biopsy is considered the gold standard for cirrhosis diagnosis. Transient elastography could offer a noninvasive alternative to biopsy to measure the stage of fibrosis, given its comparable diagnostic accuracy. Due to the earlier study period with facility limitation, we could not provide these data. In our study, 59.6\% (62/105) of patients underwent biopsy and other patients with cirrhosis were diagnosed using the clinical criteria. However, the diagnosis criteria combined with twice documented ultrasonographic evidence of liver cirrhosis with solid clinical end point (splenomegaly, ascites, hepatic encephalopathy, or varices) could complementally increase the accuracy of cirrhosis diagnosis in the current study. ${ }^{18}$ Second, this is a retrospective, single-center, observational study, which could lead to selection bias because patients with severe cirrhosis were probably not considered for treatment and therefore were not included in our study.

\section{Conclusion}

In cirrhotic $\mathrm{HCV}$-infected patients, SVR is a major predictor of HCC development, whereas APRI may be a potent predictor of HCC risk among these patients. Further studies are warranted to validate our findings and their applicability in clinical practice.

\section{Acknowledgments}

We would like to thank the nursing departments of Dalin Tzu Chi Hospital for their assistance in procuring records. The study was funded by Dalin Tzu Chi General Hospital (DTCRD99(2)-E-13).

\section{Author contributions}

Khai-Jing Ng: drafting of the manuscript; Chih-Wei Tseng: statistical analysis, material support, and drafting of the manuscript; Ting-Tsung Chang and Shu-Fen Wu: critical revision of the manuscript for important intellectual content; Shinn-Jia Tzeng: statistical analysis; Yu-Hsi Hsieh, Tsung-Hsing Hung, and Hsiang-Ting Huang: material support; Kuo-Chih Tseng: material support and critical revision of the manuscript for important intellectual content. All authors contributed toward data analysis, drafting and critically revising the paper and agree to be accountable for all aspects of the work.

\section{Disclosure}

The authors report no conflicts of interest in this work. The abstract of this paper was presented at the Asian Pacific Digestive Week, Taipei, Taiwan, 3-6 December, 2015. The abstract was published in "Poster Abstracts" in the Journal of Gastroenterology and Hepatology, special issue: Volume 30, Issue Supplement S4, December 2015. 


\section{References}

1. Herszényi L, Tulassay Z. Epidemiology of gastrointestinal and liver tumors. Eur Rev Med Pharmacol Sci. 2010;14(4):249-258.

2. Ministry of Health and Welfare. Cause of Death Statistics. 2013. Available from: http://www.mohw.gov.tw/EN/Ministry/Statistic. aspx?f_list_no=474\&fod_list_no=5044. Accessed January 19, 2015.

3. WHO. Guidelines for the Screening, Care and Treatment of Persons with Hepatitis C Infection. Geneva, Switzerland: WHO; 2014.

4. El-Serag HB. Hepatocellular carcinoma. N Engl J Med. 2011;365(12): 1118-1127.

5. Rein DB, Wittenborn JS, Weinbaum CM, Sabin M, Smith BD, Lesesne SB. Forecasting the morbidity and mortality associated with prevalent cases of pre-cirrhotic chronic hepatitis $\mathrm{C}$ in the United States. Dig Liver Dis. 2011;43(1):66-72.

6. Pearlman BL, Traub N. Sustained virologic response to antiviral therapy for chronic hepatitis $\mathrm{C}$ virus infection: a cure and so much more. Clin Infect Dis. 2011;52(7):889-900.

7. Veldt BJ, Heathcote EJ, Wedemeyer H, et al. Sustained virologic response and clinical outcomes in patients with chronic hepatitis $\mathrm{C}$ and advanced fibrosis. Ann Intern Med. 2007;147(10):677-684.

8. Bruno S, Battezzati PM, Bellati G, et al. Long-term beneficial effects in sustained responders to interferon-alfa therapy for chronic hepatitis C. $J$ Hepatol. 2001;34(5):748-755.

9. Morgan RL, Baack B, Smith BD, Yartel A, Pitasi M, Falck-Ytter Y. Eradication of hepatitis $\mathrm{C}$ virus infection and the development of hepatocellular carcinoma: a meta-analysis of observational studies. Ann Intern Med. 2013;158(5 Pt 1):329-337.

10. Hung $\mathrm{CH}$, Lee $\mathrm{CM}$, Wang JH, et al. Impact of diabetes mellitus on incidence of hepatocellular carcinoma in chronic hepatitis $\mathrm{C}$ patients treated with interferon-based antiviral therapy. Int $J$ Cancer. 2011;128(10): 2344-2352.

11. Asia-Pacific Working Party on Prevention of Hepatocellular Carcinoma. Prevention of hepatocellular carcinoma in the Asia-Pacific region: consensus statements. $J$ Gastroenterol Hepatol. 2010;25(4): $657-663$.

12. Wai CT, Greenson JK, Fontana RJ, et al. A simple noninvasive index can predict both significant fibrosis and cirrhosis in patients with chronic hepatitis C. Hepatology. 2003;38(2):518-526.

13. Vallet-Pichard A, Mallet V, Nalpas B, et al. FIB-4: an inexpensive and accurate marker of fibrosis in $\mathrm{HCV}$ infection. comparison with liver biopsy and fibrotest. Hepatology. 2007;46(1):32-36.

14. Vergniol J, Boursier J, Coutzac C, et al. Evolution of noninvasive tests of liver fibrosis is associated with prognosis in patients with chronic hepatitis C. Hepatology. 2014;60(1):65-76.

15. Yu ML, Lin SM, Lee CM, et al. A simple noninvasive index for predicting long-term outcome of chronic hepatitis $\mathrm{C}$ after interferon-based therapy. Hepatology. 2006;44(5):1086-1097.

16. Hann HW, Wan S, Lai Y, et al. Aspartate aminotransferase to platelet ratio index as a prospective predictor of hepatocellular carcinoma risk in patients with chronic hepatitis B virus infection. $J$ Gastroenterol Hepatol. 2015;30(1):131-138

17. Wu CK, Chang KC, Hung CH, et al. Dynamic alpha-fetoprotein, platelets and AST-to-platelet ratio index predict hepatocellular carcinoma in chronic hepatitis $\mathrm{C}$ patients with sustained virological response after antiviral therapy. J Antimicrob Chemother. 2016;71(7): 1943-1947.
18. Lee K, Sinn DH, Gwak GY, et al. Prediction of the risk of hepatocellular carcinoma in chronic hepatitis $\mathrm{C}$ patients after sustained virological response by aspartate aminotransferase to platelet ratio index. Gut Liver. Epub 2016 Apr 28.

19. Chung HA, Kim JH, Hwang Y, et al. Noninvasive fibrosis marker can predict recurrence of hepatocellular carcinoma after radiofrequency ablation. Saudi J Gastroenterol. 2016;22(1):57-63.

20. Fried MW. Side effects of therapy of hepatitis $\mathrm{C}$ and their management. Hepatology. 2002;36(5 Suppl 1):S237-S244.

21. Omata M, Kanda T, Yu ML, et al. APASL consensus statements and management algorithms for hepatitis $\mathrm{C}$ virus infection. Hepatol Int. 2012;6(2):409-435.

22. European Association for Study of Liver. EASL Clinical Practice Guidelines: management of hepatitis C virus infection. J Hepatol. 2014; 60(2):392-420.

23. Huang JF, Yu ML, Lee CM, et al. Sustained virological response to interferon reduces cirrhosis in chronic hepatitis C: a 1,386-patient study from Taiwan. Aliment Pharmacol Ther. 2007;25(9):1029-1037.

24. Bruix J, Sherman M; American Association for the Study of Liver Diseases. Management of hepatocellular carcinoma: an update. Hepatology. 2011;53(3):1020-1022.

25. de Lope CR, Tremosini S, Forner A, Reig M, Bruix J. Management of HCC. J Hepatol. 2012;56(Suppl 1):S75-S87.

26. Ratge D, Scheiblhuber B, Nitsche M, Knabbe C. High-speed detection of blood-borne hepatitis $\mathrm{C}$ virus RNA by single-tube real-time fluorescence reverse transcription-PCR with the LightCycler. Clin Chem. 2000;46(12):1987-1989.

27. Bullock GC, Bruns DE, Haverstick DM. Hepatitis C genotype determination by melting curve analysis with a single set of fluorescence resonance energy transfer probes. Clin Chem. 2002;48(12):2147-2154.

28. Selimovic D, El-Khattouti A, Ghozlan H, Haikel Y, Abdelkader O, Hassan M. Hepatitis C virus-related hepatocellular carcinoma: an insight into molecular mechanisms and therapeutic strategies. World $J$ Hepatol. 2012;4(12):342-355.

29. Fabregat I. Dysregulation of apoptosis in hepatocellular carcinoma cells. World J Gastroenterol. 2009;15(5):513-520.

30. But DY, Lai CL, Yuen MF. Natural history of hepatitis-related hepatocellular carcinoma. World J Gastroenterol. 2008;14(11):1652-1656.

31. Sewell JL, Stick KM, Monto A. Hepatocellular carcinoma after sustained virologic response in hepatitis $\mathrm{C}$ patients without cirrhosis on a pretreatment liver biopsy. Eur J Gastroenterol Hepatol. 2009;21(2):225-229.

32. Masuzaki R, Yoshida H, Omata M. Interferon reduces the risk of hepatocellular carcinoma in hepatitis $\mathrm{C}$ virus-related chronic hepatitis/liver cirrhosis. Oncology. 2010;78(Suppl 1):17-23.

33. Cammà $\mathrm{C}$, Giunta $\mathrm{M}$, Andreone $\mathrm{P}, \mathrm{Craxì} \mathrm{A}$. Interferon and prevention of hepatocellular carcinoma in viral cirrhosis: an evidence-based approach. J Hepatol. 2001;34(4):593-602.

34. Okuda H. Hepatocellular carcinoma development in cirrhosis. Best Pract Res Clin Gastroenterol. 2007;21(1):161-173.

35. Bruix J, Sherman M; Practice Guidelines Committee American Association for the Study of Liver Diseases. Management of hepatocellular carcinoma. Hepatology. 2005;42(5):1208-1236.

36. Huang CF, Yeh ML, Tsai PC, et al. Baseline gamma-glutamyl transferase levels strongly correlate with hepatocellular carcinoma development in non-cirrhotic patients with successful hepatitis $C$ virus eradication. $J$ Hepatol. 2014;61(1):67-74.
Clinical Interventions in Aging

\section{Publish your work in this journal}

Clinical Interventions in Aging is an international, peer-reviewed journal focusing on evidence-based reports on the value or lack thereof of treatments intended to prevent or delay the onset of maladaptive correlates of aging in human beings. This journal is indexed on PubMed Central, MedLine,
CAS, Scopus and the Elsevier Bibliographic databases. The manuscript management system is completely online and includes a very quick and fair peer-review system, which is all easy to use. Visit http://www.dovepress. com/testimonials.php to read real quotes from published authors. 